\title{
Latino ethnicity influences response to therapy
}

Sustained virological response (SVR) rates to therapy for HCV genotype 1 are lower in white Latino than in white non-Latino individuals, reported Dr Rodriguez-Torres and colleagues in their recent article in The New England Journal of Medicine. "Strategies to improve [SVRs] in Latinos are needed," the authors concluded.

Ethnicity is known to affect response to therapy in patients with HCV infection, but few relevant data are available. Latino individuals, in particular, are underrepresented in clinical trials; $\mathrm{HCV}$ infection in this group is particularly aggressive and is associated with increased mortality.

This led Rodriguez-Torres and colleagues to investigate the effect of ethnicity on response to once-weekly treatment with peginterferon a 2 a ( $180 \mu \mathrm{g} /$ week) plus ribavirin ( $1 \mathrm{~g}$ or $1.2 \mathrm{~g}$ daily) in patients with $\mathrm{HCV}$ genotype 1 . The researchers compared rates of response to therapy in white Latino individuals $(n=269)$ with those in the white non-Latino patients $(n=300)$. Only Latino individuals whose parents and grandparents spoke Spanish were eligible for inclusion in this prospective, multicenter, open-label, nonrandomized study. A significantly greater proportion of non-Latino than Latino patients (49\% versus 34\%) achieved an SVR. Furthermore, failure to detect serum HCV RNA at week 4 and throughout the treatment period occurred more frequently in non-Latino than in Latino patients. However, fewer Latino than nonLatino individuals withdrew from treatment owing to adverse events (9\% versus 14\%).

Rachel Jones

Original article Rodriguez-Torres, M. et al. Peginterferon alfa-2a and ribavirin in Latino and non-Latino whites with hepatitis C. N. Engl. J. Med. 360, 257-267 (2009). 